\title{
Numerical study of inventory management under various maintenance policies
}

\author{
Joeri Poppe ${ }^{1}$, Rob J.I. Basten ${ }^{2}$, Robert N. Boute ${ }^{1,3}$, and Marc R. Lambrecht ${ }^{1}$ \\ ${ }^{1}$ Research Center for Operations Management, University KU Leuven, Belgium \\ ${ }^{2}$ School of Industrial Engineering, TU Eindhoven, Netherlands \\ ${ }^{3}$ Technology $\& 3$ Operations Management Area, Vlerick Business School, Belgium
}

June 12, 2017

\begin{abstract}
Capital assets, such as manufacturing equipment, require maintenance to remain functioning. Maintenance can be performed when a component breaks down and needs replacement (i.e., corrective maintenance), or the maintenance and part replacement can be performed preventively. Preventive maintenance can be planned on a periodic basis (periodic maintenance), or it can be triggered by a certain monitored condition (condition-based maintenance). Preventive maintenance policies are gaining traction in the business world, but for many companies it is unclear what their impact is on the resulting inventory requirements for the spare parts that are used for the maintenance interventions. We study the impact of the maintenance policy on the inventory requirements and the corresponding costs for a setting that is realistic at an OEM in the compressed air industry. Preventive policies increase the total demand for spare parts compared to corrective maintenance, since the former do not exploit the entire useful life of the components. This leads to higher inventory requirements. At the same time, the preventive policies inhibit advance demand information, as the interventions, and correspondingly the spare parts demands, are planned in advance. Using a simulation study, we show that by using this advance demand information in managing the spare part inventory, the increase in inventory requirements of preventive maintenance policies can to a large extent be offset; for condition-based maintenance, we find that inventories can even be lower compared to corrective maintenance, provided that the advance demand information is used correctly when managing inventories. Our analysis sheds light on the behaviour of the inventory related costs under various maintenance policies.
\end{abstract}

Keywords: servitisation, maintenance, condition-based maintenance, spare parts inventory management, advance demand information

\section{Introduction}

Modern society depends to a large extent on the functioning of capital assets, which are expensive systems having a long life time. Examples are the trains and railroads that are used to transport people and freight, the equipment that is used in the process industry, and the machines that are used to 
manufacture the goods that we use daily. These assets require a lot of maintenance throughout their life cycle. Van Dongen [30] gives various examples of systems incurring more maintenance costs throughout their life cycles than their initial acquisition costs; e.g., for rolling stock and the joint strike fighter this is about twice as much.

Some users maintain their assets themselves. Examples are most national railway operators and defense organisations. Others outsource all or some maintenance to the original equipment manufacturers or system integrators (we will refer to both as OEMs) or to third parties. One reason to do so is that assets are often so complex that it is hard for users to maintain them: they lack the specific knowledge and skills that are required to perform the more complicated maintenance tasks. For the OEMs, it is in their interest to take over the maintenance, and to deliver other services, since these after sales activities generate a more steady revenue stream with profit margins that are often more rewarding than the sales of the assets themselves. This trend is called servitisation. In its extreme form, users are not even acquiring the asset anymore; they only buy the use of it ('power by the hour'). We refer to Basten and Van Houtum [2] and the references therein for a further discussion of the different types of users and the reasons to outsource maintenance. In the remainder of this paper, we will take the perspective of an OEM, who is in charge of the maintenance of the installed base of machines at its customers' premises. The reason is that our work is motivated by, and validated at an OEM in the compressed air, generator and pump industry. The OEM deploys a preventive maintenance strategy on the majority of its components and started to implement condition-based maintenance on a selected number of components. However, the findings are also useful for users maintaining their own assets.

To be able to perform maintenance, various resources are required: spare parts, trained personnel, facilities, tools, and test equipment [15]. If these resources are not available, maintenance cannot be performed. We focus in this paper on the relation between the maintenance policy and the spare parts inventory control policy. The maintenance policies that we will use throughout this paper and that are most frequently encountered, both in academia and in practice, are: corrective maintenance $(\mathrm{CM})$, periodic maintenance $(\mathrm{PM})$, and condition-based maintenance $(\mathrm{CBM})$, with the latter two being preventive maintenance policies. (We come back to these policies in Section 2 and Section 3.) If corrective maintenance is applied, with maintenance being performed upon failure, then the demand for spare parts depends on the failure behaviour of the assets only. This is different if maintenance is performed preventively. For instance, in case of periodic maintenance, it is a combination of a deterministic demand stream of spare parts for the PM interventions, and some random demands in case a component breaks down prior to the preventive maintenance. The stochastic nature of the degradation behaviour, the maintenance policy that is applied, and the resulting degree of advance demand information determine the requirements for the inventory control policy.

Still, in practice, it often happens that if some demands for spare parts are planned, while others are unplanned, they are all treated as being the same, i.e., unpredictable without taking into account the maintenance policy. In other words, the advance demand information generated by the maintenance policy is not used to manage inventories. We noticed this at several OEMs with whom we collaborate, but it is also noticed by others [see, e.g., 8]. Therefore, our first contribution is that we show how the standard inventory control policies that are used in practice can be improved, by leveraging the advance demand information generated by the maintenance policy. In other words, we show the inventory cost reductions that can be obtained by not simply treating all demands as being random and unplanned.

Our second contribution is that we compare the inventory costs of the three mentioned maintenance 
policies combined with their inventory control policies. This results in a more thorough comparison in which not only maintenance and downtime costs, but also the inventory costs for spare parts are included. To the best of our knowledge, such a comparison has not been performed in the literature; we come back to the existing comparison studies in Section 2.

We achieve these contributions using a numerical simulation study. We focus on the maintenance policy and spare parts for one component, and we assume that this component is critical, so that failure of the component causes the machine to stop. We analyse the inventory performance of the different maintenance policies under a variety of settings, e.g., failure behaviour of components and size of the installed base. We focus especially on CBM, since that policy is getting more popular in practice and in the literature [see, e.g., 34, and the references therein]. One reason for its popularity is that monitoring and storing data becomes cheaper and big data technology allows for better analyses. Another reason is that when looking at the maintenance and downtime costs only, this policy is typically the most cost-effective one [see, e.g., 22].

The remainder of this paper is organised as follows. Section 2 reviews the related literature. In Section 3 we discuss the maintenance policies that we consider in our numerical study. In Section 4 we introduce our model, the assumptions that we make and the notation that we use. In this section we also address how the inventory policy can be adapted based on the maintenance policy in use. We present our extensive numerical study in Section 5. Section 6 concludes.

\section{Related literature}

We first discuss some literature related to the degradation and failure behaviour of components. We then discuss literature related to the maintenance policies considered in this paper and its related spare parts inventory control, with specific focus on the use of advance demand information. We conclude with some comparison studies that are related to ours.

When components are in use, they degrade, resulting at some point in a failure. There are many ways in which degradation behaviour and thus failure behaviour can be modelled. We discuss a few well known examples. The delay time model (DTM) was introduced by $[5,6]$ and is discussed more recently, for example, by Wang [36]. In the model, it is assumed that a component can be in three states: good, defect and failed. The time that the component remains good, the time-to-defect, has a certain distribution, while the time that the component remains in the defect state, the delay time, has another distribution. This model is especially useful when the degradation behaviour is monitored using inspections, and the goal is typically to optimise the inspection interval. The time-to-failure of the component is the convolution of the time-to-defect and the delay time. A somewhat related model that can be used to model degradation behaviour is the Markov chain, in which there can be any number of states and a component remains in a state an exponentially distributed amount of time. If the sojourn time in each state is equal, the time-to-failure is Erlang distributed. If there is only one state that can be distinguished, the time-to-failure is exponentially distributed. This is often assumed for electronic components.

The degradation can also in some cases be tracked on a continuous scale. In that case, the Gamma process can be used to model the degradation behaviour. It is a process with independent gamma distributed increments, which means that degradation can only increase. Van Noortwijk [33] gives an excellent review of the Gamma process applied to maintenance. We use a special case of the random coefficient model. In this model, degradation follows a certain function with known and unknown parameters, or 
coefficients. If all parameters are known, the degradation path is deterministic. The original application of random coefficient models is to estimate the time-to-failure distribution based on degradation data [19]. The special case that we consider is linear with one unknown parameter. This parameter itself is Weibull distributed, which means that the time to failure is also Weibull distributed. It allows us to focus on the interesting insights, without using too complicated models.

Given the degradation and failure behaviour of a component, it can be determined when to perform maintenance. This means that it should be decided which maintenance policy to use, and how to set its parameters. We consider three different maintenance policies, which we discuss in more detail in Section 3. One of them is the policy of corrective maintenance, which means that maintenance is performed upon failure. The other two policies are periodic maintenance and condition based maintenance, which are both preventive maintenance policies. This means that maintenance is planned some time in advance, in order to prevent failures. This results in so-called advance demand information [ADI; see, e.g., 9, 12]. There are many overviews of maintenance policies, for instance, Wang [35], Tinga [27, 28], Van Horenbeek et al. [34], or Goossens and Basten [11].

A maintenance intervention may mean various things, but we assume that it means a replacement of a failed component by a spare part. For extensive treatments on spare parts inventory control, typically not considering the maintenance policy, we refer to the books by Sherbrooke [24], Muckstadt [20], Van Houtum and Kranenburg [32] or the review paper by Basten and Van Houtum [2]. The literature is extensive, and we do include an exhaustive list. Different assumptions are made in the literature, for example on who owns the spare parts and performs maintenance (as discussed in Section 1). If an OEM owns spare parts to service many customers, there are pooling effects that lower the total required number of spare parts compared with the situation that each customer owns its own spare parts. There exist also situations where customers join forces and pool their spare parts, which is modeled, for example, in [16]. From a modeling point of view, there is often no real difference between components that are repairable and those that are consumable. In both cases there is an agreed lead time, with a repair shop or a supplier, respectively, that is often modelled as being deterministic because of the agreement. In case of a stock out, demands can be backordered, or an emergency procedure may be used to supply a part quickly from another location. This is an important assumption that makes the analysis of models quite different (a good discussion can be found in [3]). Based on the situation at the OEM with whom we collaborate, we assume backordering.

One topic in spare parts inventory control that we treat in more detail is the usage of advance demand information (ADI). The seminal paper on ADI is that by Hariharan and Zipkin [12], but not specifically in spare parts inventory control. Whereas Hariharan and Zipkin [12] consider perfect ADI, it may also be imperfect. For example, Topan et al. [29] consider three types of imperfectness: The demand lead time may be stochastic, a demand that is preceded by ADI may not materialise, and a demand may materialise that is not preceded by ADI. Topan et al. [29] give the setting of spare parts inventory control and condition monitoring as one example where their model applies. However, they do not explicitly model the degradation behavior of the critical components to derive ADI. Examples of papers that do so are Deshpande et al. [7], Godoy et al. [10], Li and Ryan [17] and Louit et al. [18].

There exist some papers in which the cost performance of maintenance policies are compared. For example, Camci [4] uses a theoretical case to compare the costs resulting from applying a corrective, periodic or condition-based maintenance policy. Tinga [27] proposes two maintenance policies and compares them with some other policies on a case of a gas turbine blade. Van Horenbeek and Pintelon [31] propose a 


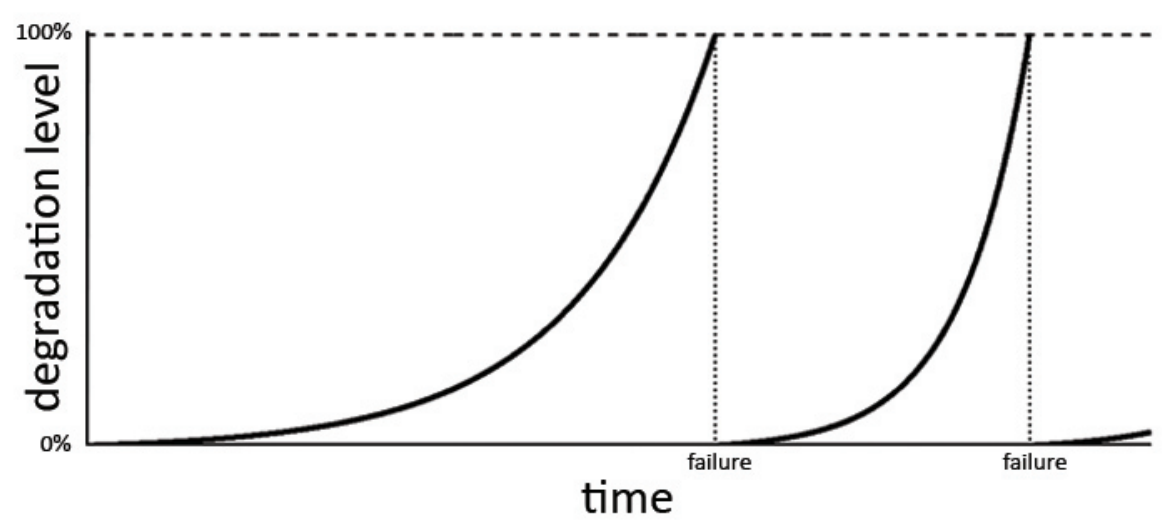

Figure 1: The corrective maintenance policy $(\mathrm{CM})$

condition-based maintenance policy for a multi-item system and then compare numerically a few periodic and condition-based maintenance policies. Finally, Srinivasan and Parlikad [25] compare the costs for maintenance (including downtime costs) when condition monitoring capabilities are installed with the case in which they are not installed. However, in this literature, costs for spare parts inventories are not incorporated. Although the importance of the joint optimisation of maintenance and inventory policies has been raised before [see, e.g., 23, 26, 34, 37], to the best of our knowledge, an inventory cost comparison of multiple maintenance policies combined with inventory control policies has not been performed in the literature; we believe to be the first to do so.

\section{Maintenance policies}

In our study we consider three different maintenance policies. The simplest policy is the corrective maintenance (CM) policy, also called failure-based maintenance. Maintenance is then executed upon failure of a component, i.e., when a component does not fulfill its intended purpose (properly) anymore. Fig. 1 illustrates this policy (without loss of generality, we assume that the degradation level goes from $0 \%$ to $100 \%$, and $100 \%$ equals failure of the component). Next to its simplicity, the main advantage of this policy is that no remaining useful life of components is wasted, meaning that the minimal number of parts is used and the minimal number of maintenance actions is required. The key drawback of the CM policy is that when maintenance is required, the resources have not been scheduled to be sent to the machine yet, which means that costly emergency measures may be required and the machine will be down waiting for the resources, incurring high downtime costs.

To prevent this, a periodic maintenance (PM) policy can be adopted. A preventive maintenance intervention is then planned, for example, every so many running hours of the machine, i.e., use-based maintenance, or after a certain calendar time, i.e., time-based maintenance. We denote this periodic maintenance interval by $\tau$. Fig. 2 illustrates this policy, specifically a block replacement policy, i.e., a policy in which the planned maintenance interventions are not rescheduled if a failure occurs within a certain periodic maintenance interval. When the interventions are planned in advance, its resource planning can be anticipated and therefore the intervention cost is usually lower than in a CM policy. The main drawback of PM is that the lifetime of components is not fully utilized, meaning that a component is replaced while it is still functioning (i.e., the component is 'over-serviced'). To decrease the effect of 


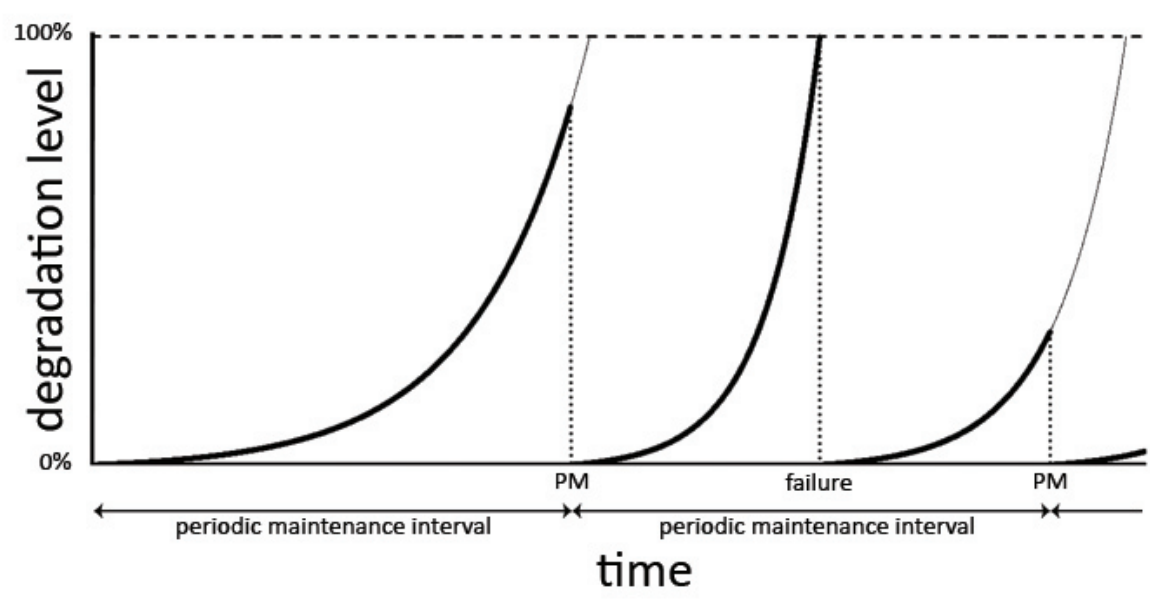

Figure 2: The periodic maintenance policy (PM)

this drawback, the maintenance interval $\tau$ may be extended, reducing the number of PM interventions. However, that also means that more parts will fail before the scheduled replacement occurs, resulting in more corrective maintenance interventions (observe that as $\tau$ increases to $\infty$, the PM policy reduces to a CM policy). This is the trade-off when determining the optimal maintenance interval $\tau^{*}$ in this policy. Optimisation of a PM policy has first been treated by Barlow and Hunter [1]; nowadays, any standard textbook on reliability engineering or maintenance optimisation treats this topic [e.g., 14, 21].

In contrast to the corrective maintenance policy, the PM interventions are planned and known (often a long time) in advance. This results in advance demand information [ADI; see, e.g., 9, 12]. This means that the demand for a spare part is announced some time in advance of the actual spare part consumption, defined as a demand lead time, $L_{\mathrm{D}}$ [see, e.g., 12]. Defining the inventory replenishment lead time as the supply lead time $L_{\mathrm{S}}$, then there is no benefit of knowing the upcoming demand earlier than $L_{\mathrm{S}}$ periods in advance. Hence, the demand lead time $L_{\mathrm{D}}=L_{\mathrm{S}}$ when using a block replacement policy.

Under a PM policy, the length of the maintenance interval $\tau^{*}$ can be optimised (based on experience or numerically) with the degradation behaviour of similar machines. However, the maintenance interventions themselves are planned independent of the actual degradation state of the machine. This is different in a condition-based maintenance (CBM) policy, where the actual condition, i.e., level of degradation, is measured continuously and determines when to replace the component. For example, a vibration level is measured or the number of metal parts in oil. Typically, CBM operates with a threshold value on the level of degradation, defined as a percentage of the critical degradation level at which the component fails. This intervention threshold, which we denote by $\varepsilon$, should give the company enough time to intervene before a failure occurs. The time period to plan this CBM intervention, is defined as the CBM planning period, $t_{\mathrm{p}}$. In this time period, a failure may still occur, in which case a CM intervention is performed instead of the planned CBM intervention. The lower the value of $\varepsilon$, the lower the likelihood of failure, but the higher the number of CBM interventions and spare part usage. Note that if $t_{\mathrm{p}}$ is very short, then the degradation threshold may be set close to the failure level, in which case the CBM policy becomes increasingly similar to the CM policy. Fig. 3 illustrates a CBM policy.

As the intervention threshold announces an upcoming maintenance intervention, and thus a spare part replacement, it also generates ADI. If no failure occurs during the CBM planning period, the demand lead 


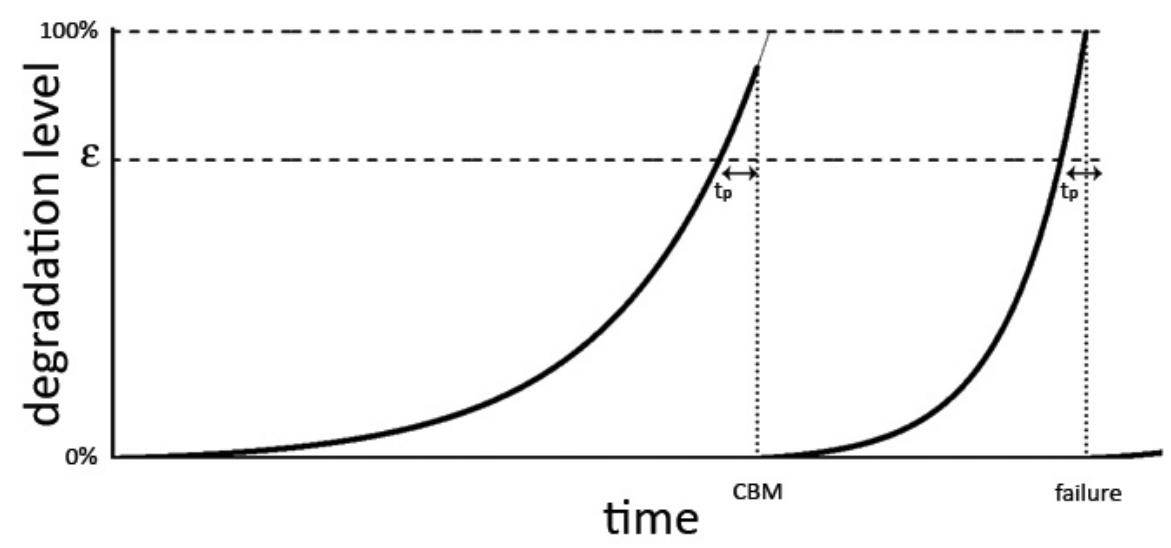

Figure 3: The condition-based maintenance policy (CBM)

time $L_{\mathrm{D}}=t_{\mathrm{p}}$. In case the critical monitored component fails before the extra maintenance intervention is performed (i.e., within the planning period $t_{\mathrm{p}}$ ), then $L_{\mathrm{D}}<t_{\mathrm{p}}$. In other words, the demand lead time $L_{\mathrm{D}}$ in CBM is stochastic and at most $t_{\mathrm{p}}$. In Section 4 we quantify its expected value.

As stated by Hariharan and Zipkin [12], "the effect of a demand lead time on overall system performance is precisely the same as a corresponding reduction in the supply lead time [...] A supply lead time increases uncertainty about the future (i.e., it increases the risk period in which a stockout may occur), while a demand lead time reduces it". This is exactly what we exploit in our simulation.

\section{Model}

In this section, we describe the model that we use to investigate the impact of the maintenance policy on the spare parts inventory control policy and the resulting total costs. Section 4.1 introduces the notation and assumptions, and Section 4.2 is devoted to the optimisation model itself. We then give the exact model for each of the three maintenance policies that we consider in Sections 4.3, 4.4, and 4.5, respectively.

\subsection{Notation and assumptions}

We provide an overview of the notation that we introduce in Table 1. We consider an installed base of $M$ machines, with each machine running for $R$ running hours annually. We assume that $R$ is smaller than the total number of hours in one year (i.e., $R=2,000$, while $365 \times 24=8,760$ ), so that preventive maintenance can be planned when the machine is not running. We analyse the long-run performance of the system.

We consider a setting with a large number of components and focus on a single critical component that is present in each of the $M$ machines in the installed base. We assume that this component has one failure mode. A maintenance action corresponds to a replacement of the component. Upon replacement, we assume the critical component to be as-good-as-new. We use the two-parameter Weibull distribution to characterise the stochastic failure behaviour of this component. This distribution is commonly used 


\begin{tabular}{ll}
\hline Input parameters \\
\hline$M$ & Number of machines in the installed base (IB) \\
$R$ & Annual number of running hours per machine \\
$\alpha$ & Scale parameter of the failure distribution of the component (characteristic life) \\
$\beta$ & Shape parameter of the failure distribution of the component \\
$C_{\mathrm{h}}$ & Annual holding cost per unit \\
$K$ & Fixed cost per replenishment order \\
$C_{\mathrm{CM}}$ & Cost of a CM intervention \\
$C_{\mathrm{PM}}$ & Cost of a PM intervention \\
$C_{\mathrm{CBM}}$ & Cost of a CBM intervention \\
$t_{\mathrm{p}}$ & CBM planning period \\
$L_{\mathrm{S}}$ & Supply lead time \\
$F_{\mathrm{r}}$ & Required fill rate \\
\hline Decision variables \\
\hline$s$ & Reorder point \\
$S$ & Order-up-to level \\
$Q$ & Order quantity \\
$\tau$ & Maintenance interval in running hours under a PM policy \\
$\varepsilon$ & Intervention threshold (as a percentage of the critical degradation level at which the \\
& component fails) under a CBM policy \\
\hline Other parameters \\
\hline$P$ & Maintenance policy \\
$t$ & Time since last maintenance intervention \\
$T$ & Time to failure of a component \\
$L_{\mathrm{D}}$ & Demand Lead time \\
$F_{\mathrm{a}}$ & Achieved fill rate \\
$I$ & On hand inventory of spare parts \\
$D$ & Annual demand for spare parts \\
$\mathcal{Y}_{\tau}$ & Number of failures between two PM interventions in the interval $\tau$ \\
\hline Functions & \\
\hline$I C(P)$ & Inventory costs of maintenance policy $P$ \\
$M C(P)$ & Maintenance intervention costs of maintenance policy $P$ \\
\hline &
\end{tabular}

due to its flexibility to model a variety of realistic failure behaviours. We use the stochastic variable $T \geq 0$ to denote the time to failure of a component. With $t \geq 0$ the time since the last maintenance intervention, $T$ has the following probability density function $f(t)$, cumulative distribution function $F(t)$ and reliability function $R(t)$ :

$$
\begin{aligned}
& f(t)=\frac{\beta}{\alpha}\left(\frac{t}{\alpha}\right)^{\beta-1} \exp \left[-\left(\frac{t}{\alpha}\right)^{\beta}\right], \\
& F(t)=1-\exp \left[-\left(\frac{t}{\alpha}\right)^{\beta}\right], \text { and } \\
& R(t)=1-F(t)=\exp \left[-\left(\frac{t}{\alpha}\right)^{\beta}\right]
\end{aligned}
$$


The expected time to failure $\mathbb{E}\{T\}$ is then given by:

$$
\mathbb{E}\{T\}=\alpha \Gamma\left(1+\frac{1}{\beta}\right)
$$

with $\Gamma(*)$ denoting the Gamma function. The parameter $\alpha$ (denoting the characteristic life parameter) scales the probability distribution function: a small value of $\alpha$ means that on average the component will fail fast, indicating a short expected time to failure $\mathbb{E}\{T\}$. The shape parameter $\beta$ determines the shape of the Weibull distribution. A value $\beta>1$ is most common in practice and means that the component exhibits an increasing failure rate. For $\beta<1$, the failure rate is decreasing. If $\beta=1$, then the failure probability is constant over time and the Weibull distribution reduces to the (negative) exponential distribution. In this paper we use the common assumption of $\beta>1$, since periodic maintenance has no use otherwise. Under this assumption, a larger $\beta$-value indicates a smaller variance of the failure time. Furthermore, we consider a linearly increasing degradation behaviour.

We denote the maintenance policy on our focal component by $P$. As mentioned in Section 3 , we consider three maintenance policies: Corrective Maintenance, Periodic Maintenance (with block replacements), and Condition-Based Maintenance, i.e., $P \in\{\mathrm{CM}, \mathrm{PM}, \mathrm{CBM}\}$. A maintenance intervention upon failure of the component, which can happen under each of the three policies, is denoted as a 'CM intervention' at cost $C_{\mathrm{CM}}$. So-called 'PM interventions' cost $C_{\mathrm{PM}}$ and 'CBM interventions' cost $C_{\mathrm{CBM}}$. The cost of a maintenance intervention includes the costs for the spare part that is used, which is a remanufacturing (or refurbishment) cost for a repairable part or the purchasing cost for a consumable part, plus costs to get the parts from the stock point to the maintenance location. The intervention cost further includes the costs to get, employ, and return a service engineer, tooling, and further materials to the maintenance location. These costs will be lower if the intervention can be planned in advance. In case of an unplanned $\mathrm{CM}$ intervention, also some downtime costs (outage costs due to loss of productivity) are included, and possibly consequential damage on other parts of the machine. As a result, we assume $0 \leq C_{\mathrm{PM}} \leq$ $C_{\mathrm{CBM}} \leq C_{\mathrm{CM}}$. These cost parameters represent the expected maintenance cost of a single intervention. Depending on the characteristics of the component, these intervention costs can differ. For instance, for different parts in a gas turbine, the cost parameters for different maintenance policies will be different. In our numerical study we will make this distinction explicitly. We acknowledge that the intervention cost itself may display some uncertainty - however, including the uncertainty of these parameters by modelling the estimation errors would go beyond the purpose of this paper. We refer to Hill [13], who addresses estimation uncertainty in inventory models that build on Bayesian models.

We further assume an annual holding $\operatorname{cost} C_{\mathrm{h}}$ per unit in inventory and a fixed order cost $K$ to place a replenishment order, independent of the order quantity. We consider a continuous review system with an $(s, S)$ policy: When the inventory position hits the reorder point $s$, a replenishment order is placed to raise the inventory position to the order-up-to-level $S$. The order is received after the constant supply lead time $L_{\mathrm{S}}$. We assume a required fill rate of $F_{\mathrm{r}}$, imposed by the service contract. When a stock-out occurs, the units are backlogged and fulfilled after replenishment.

It is worthwhile to elaborate on the inventory control policy under the various maintenance policies. In case of CM, all demands for spare parts are stochastic. There is no advance demand information and a classical $(s, S)$ policy is implemented. In case of PM and CBM, advance demand information exists and could be exploited.

When the demand of a spare is announced $L_{\mathrm{D}}$ time in advance (this is the spare part 'order'), we reduce 
the inventory position (parts on stock plus parts on order), while we use a first-come-first-served policy on the actual spare part consumption at the moment a maintenance is performed. In this way, the demand lead time allows to anticipate and consequently reduce the risk period.

\subsection{Model formulation}

Given a maintenance policy $P \in\{\mathrm{CM}, \mathrm{PM}, \mathrm{CBM}\}$ on our component, we aim to find the lowest expected annual costs, while achieving the target fill rate $F_{\mathrm{a}} \geq F_{\mathrm{r}}$, with $F_{\mathrm{a}}$ the achieved fill rate defined as the number of times a spare part was in stock upon request divided by the total number of spare parts demanded. The annual costs consist of the component's inventory costs and maintenance costs; inventory costs relate to the inventory holding and ordering costs, while maintenance costs consist of the cost per maintenance intervention times the annual number of maintenance interventions. As discussed above, the cost of a maintenance intervention (CM, PM, or CBM) is composed of all cost factors related to the maintenance and potential failure of the component, including consequential damage and downtime costs. We define the cost functions for the component's inventory and maintenance costs as $I C(P)$ and $M C(P)$, respectively. Due to the stochastic nature of the component's failure behaviour, these cost functions are stochastic as well. We minimise the long-run average total costs by optimising the maintenance policy (i.e., determining the optimal value of $\tau$ in PM, and of $\varepsilon$ in CBM) and the inventory control parameters $s$ and $S$ for the spare part requirements corresponding to that maintenance policy. This results in the following optimisation problem $(O)$ :

$$
\begin{aligned}
\underset{s, S, \tau, \varepsilon}{\operatorname{minimise}} & I C(P)+M C(P) \\
\text { subject to } & F_{\mathrm{a}} \geq F_{\mathrm{r}} .
\end{aligned}
$$

As not all terms in (5) are analytically tractable due to its stochastic nature, we rely on simulation optimisation to determine the optimal parameters of the $(s, S)$ policy, as well as the values of $\tau^{*}$ and $\varepsilon^{*}$ when respectively PM or CBM is adopted. In the next sections we outline how the optimisation problem $(O)$ is specified for each maintenance policy and provide analytical expressions for parts of the objective function in (5).

\subsection{Corrective Maintenance}

When a CM policy is in place, the annual demand for spare parts, $D$, coincides with the annual number of CM interventions, which is equal to the annual number of failures. Therefore, the spare part demand per year is stochastic. The expected demand equals $\mathbb{E}\{D\}=\frac{M \times R}{\mathbb{E}\{T\}}$, with $M$ the number of machines in the installed base, $R$ the annual number of running hours per machine, and $T$ the stochastic time to failure. The ordering costs are given by $K \frac{\mathbb{E}\{D\}}{S-s}$, and the expected annual maintenance costs equal $M C(\mathrm{CM})=C_{\mathrm{CM}} \times \mathbb{E}\{D\}$. Only the expected on hand inventory, $\mathbb{E}\{I\}$, to meet the target fill rate $F_{\mathrm{r}}$, cannot be given analytically, and is to be simulated for a given set of $(s, S)$ parameters. In order to obtain the optimal set of $(s, S)$ parameters we make use of the distribution of the demand during lead time. This lead time demand distribution is obtained through simulation. 
For corrective maintenance, Problem $(O)$ can thus be written as:

$$
\begin{array}{ll}
\underset{s, S}{\operatorname{minimise}} & \left(C_{\mathrm{h}} \times \mathbb{E}\{I\}+K \frac{M \times R}{\mathbb{E}\{T\} \times(S-s)}\right)+\left(C_{\mathrm{CM}} \frac{M \times R}{\mathbb{E}\{T\}}\right) \\
\text { subject to } & F_{\mathrm{a}} \geq F_{\mathrm{r}} .
\end{array}
$$

\subsection{Periodic Maintenance}

Under a PM policy, two types of maintenance interventions are performed. First, a PM intervention is executed on the basis of a fixed maintenance interval $\tau$. These PM interventions generate a demand for spare parts, $D_{\mathrm{PM}}$. Second, if a failure cannot be prevented, a CM intervention is performed to replace the component upon failure. Let $D_{\mathrm{CM}}$ denote the stochastic demand for spares for these CM interventions. When a failure occurs, the PM schedule, and thus $D_{\mathrm{PM}}$, remains unaffected (see also Fig. 2). Under a PM policy the total annual expected spare part demand for our component is then given by $\mathbb{E}\{D\}=\mathbb{E}\left\{D_{\mathrm{PM}}\right\}+\mathbb{E}\left\{D_{\mathrm{CM}}\right\}=\frac{M \times R}{\tau}+\frac{M \times R \times \mathbb{E}\{\mathcal{Y}\}_{\tau}}{\tau}$, with $\mathbb{E}\{\mathcal{Y}\}_{\tau}$ the expected number of failures between two subsequent PM interventions in the interval $\tau$. $\mathbb{E}\{\mathcal{Y}\}_{\tau}$ can be calculated as:

$$
\begin{aligned}
\mathbb{E}\{\mathcal{Y}\}_{\tau} & =\int_{t_{1}=0}^{\tau} f\left(t_{1}\right) \mathrm{d} t_{1}+\int_{t_{1}=0}^{\tau} \int_{t_{2}=0}^{\left(\tau-t_{1}\right)} f\left(t_{2}\right) \mathrm{d} t_{2} f\left(t_{1}\right) \mathrm{d} t_{1} \\
& +\sum_{i=3}^{\infty} \int_{t_{1}=0}^{\tau} \int_{t_{2}=0}^{\left(\tau-t_{1}\right)} \ldots \int_{t_{i}=0}^{\left(\tau-t_{1} \ldots-t_{i-1}\right)} f\left(t_{i}\right) \mathrm{d} t_{i} \ldots f\left(t_{2}\right) \mathrm{d} t_{2} f\left(t_{1}\right) \mathrm{d} t_{1},
\end{aligned}
$$

where $t_{1}$ is the time between the PM intervention and the first failure (and thus CM intervention), $t_{2}$ is the time between the first and second failure, etc. The intuition behind Equation (6) is the following: its first term defines the probability that at least one failure will occur during $\tau$ time units, the second term defines the probability that at least two failures occur during $\tau$ time units, etc. For instance, the probability of at least two failures during $\tau$ time units, corresponds with the situation when the first failure occurs after $t_{1}<\tau$ time units and the second failure occurs after $t_{2}<\tau-t_{1}$ time units, so that the second failure occurs before $\tau$ time units have passed, i.e., before the next PM intervention.

The PM policy is now optimised by jointly determining the values of $\tau, s$ and $S$ that minimise the total costs. Problem $(O)$ can thus be formulated as:

$$
\begin{aligned}
& \underset{\tau, s, S}{\operatorname{minimise}}\left(C_{\mathrm{h}} \times \mathbb{E}\{I\}+K \frac{\mathbb{E}\{D\}}{S-s}\right)+\left(C_{\mathrm{PM}} \times \mathbb{E}\left\{D_{\mathrm{PM}}\right\}+C_{\mathrm{CM}} \times \mathbb{E}\left\{D_{\mathrm{CM}}\right\}\right) \\
& \text { subject to } \quad F_{\mathrm{a}} \geq F_{\mathrm{r}} .
\end{aligned}
$$

Notice that the length of the maintenance interval $\tau$ influences both the maintenance intervention costs, as well as the inventory costs. Similar to the CM policy, all terms except $\mathbb{E}\{I\}$ are analytically tractable. This is why we rely on simulation to evaluate the objective function and take the stochastic character of the failure behaviour into account.

Up till now we ignored the fact that $D_{\mathrm{PM}}$ can be managed differently given the fact that PM interventions are planned in advance. We can leverage this ADI to improve the predictability of the PM interventions. The demand lead time for PM interventions is $L_{\mathrm{D}}=L_{\mathrm{S}}$, which allows to replenish units for the periodic preventive maintenance $L_{\mathrm{S}}$ periods ahead of time. The demand $D_{\mathrm{PM}}$ can then be replenished without 
the necessity of safety stocks. Only the demand for $\mathrm{CM}$ interventions, $D_{\mathrm{CM}}$, remains unpredictable as it does not inhibit ADI, and requires safety stocks.

\subsection{Condition-Based Maintenance}

Recently, monitoring degradation levels has become cheaper, and big data analytics are progressing rapidly, which lead to condition-based maintenance (CBM) gaining interest to both practitioners as well as researchers [see 14]. Our case company installed sensors on the equipment sold and is able to monitor continuously the degradation level of a component or system. The degradation curve and the associated intervention threshold, $\varepsilon$, are at the core of our CBM model. Under a CBM policy with continuous monitoring, there are also two types of maintenance interventions: if the threshold degradation value $\varepsilon$ is reached, a CBM maintenance intervention is planned and executed after the CBM planning period $t_{\mathrm{p}}$. In case the component fails during this planning period, a CM intervention is required and the planned CBM intervention will not take place (see Fig. 3).

When the entire spare part demand for the component under focus, $D=D_{\mathrm{CBM}}+D_{\mathrm{CM}}$, is managed by an $(s, S)$ maintenance policy, the objective function is:

$$
\begin{aligned}
& \underset{\varepsilon, s, S}{\operatorname{minimise}}\left(C_{\mathrm{h}} \times \mathbb{E}\{I\}+K \frac{\mathbb{E}\{D\}}{(S-s)}\right)+\left(C_{\mathrm{CBM}} \times \mathbb{E}\left\{D_{\mathrm{CBM}}\right\}+C_{\mathrm{CM}} \times \mathbb{E}\left\{D_{\mathrm{CM}}\right\}\right) \\
& \text { subject to } \quad F_{\mathrm{a}} \geq F_{\mathrm{r}} .
\end{aligned}
$$

The number of interventions per year $D$ is stochastic; its expected value equals $\mathbb{E}\{D\}=\frac{M \times R}{\mathbb{E}\left\{T_{\mathrm{c}}\right\}}$, with $\mathbb{E}\left\{T_{\mathrm{c}}\right\}$ the average maintenance cycle time, being the average time between two maintenance interventions, independent of the type of intervention. The expected number of respectively CBM and CM interventions are given by $\mathbb{E}\left\{D_{\mathrm{CBM}}\right\}=\mathbb{P}\{\mathrm{CBM}\} \times \mathbb{E}\{D\}$ and $\mathbb{E}\left\{D_{\mathrm{CM}}\right\}=\mathbb{P}\{\mathrm{CM}\} \times \mathbb{E}\{D\}$, with $\mathbb{P}\{\mathrm{CBM}\}$ the probability of a $\mathrm{CBM}$ intervention and $\mathbb{P}\{\mathrm{CM}\}$ the probability of a $\mathrm{CM}$ intervention. The probabilities $\mathbb{P}\{\mathrm{CBM}\}$ and $\mathbb{P}\{\mathrm{CM}\}$, and the average maintenance cycle time $\mathbb{E}\left\{T_{\mathrm{c}}\right\}$ can be analytically derived as follows. We divide the lifetime $t$ of the monitored component into two parts,

$$
t=t_{\varepsilon}+t_{\varepsilon, \mathrm{F}}
$$

where $t_{\varepsilon}$ defines the degradation time from replacement to the intervention threshold $\varepsilon$, and $t_{\varepsilon, \mathrm{F}}$ the degradation time between $\varepsilon$ and failure of the monitored component (in this time window, a failure is prevented if $\left.t_{\mathrm{p}} \leq t_{\varepsilon, \mathrm{F}}\right)$. To define their respective probability density function, denoted by $f^{\prime}\left(t_{\varepsilon}\right)$ and $f^{\prime \prime}\left(t_{\varepsilon, \mathrm{F}}\right)$, recall that we defined (without loss of generality) failure upon $100 \%$ degradation, and as-goodas-new ( $0 \%$ degradation) upon replacement, and the failure behaviour following a two-parameter Weibull distribution described by Eq. (1). Then, as $t_{\varepsilon}$ and $t_{\varepsilon, F}$ are proportional to the lifetime $t$ under a linear degradation process, we can obtain the probability density function of the degradation time between two degradation levels by multiplying the degree of degradation between these levels (which is $\varepsilon$ for $t_{\varepsilon}$ and $(1-\varepsilon)$ for $t_{\varepsilon, F}$, respectively) with the scale parameter $\alpha$. Note that a similar (albeit slightly more complex) analysis can be done for other degradation processes, e.g., exponential degradation. This leads 
to:

$$
\begin{aligned}
f^{\prime}\left(t_{\varepsilon}\right) & =\frac{\beta}{\varepsilon \alpha}\left(\frac{t_{\varepsilon}}{\varepsilon \alpha}\right)^{\beta-1} \exp \left[-\left(\frac{t_{\varepsilon}}{\varepsilon \alpha}\right)^{\beta}\right], \text { and } \\
f^{\prime \prime}\left(t_{\varepsilon, \mathrm{F}}\right) & =\frac{\beta}{(1-\varepsilon) \alpha}\left(\frac{t_{\varepsilon, \mathrm{F}}}{(1-\varepsilon) \alpha}\right)^{\beta-1} \exp \left[-\left(\frac{t}{(1-\varepsilon) \alpha}\right)^{\beta}\right] .
\end{aligned}
$$

In a similar way, we can derive the corresponding cumulative failure and reliability functions $F^{\prime}\left(t_{\varepsilon}\right)$, $F^{\prime \prime}\left(t_{\varepsilon, \mathrm{F}}\right), R^{\prime}\left(t_{\varepsilon}\right)$ and $R^{\prime \prime}\left(t_{\varepsilon, \mathrm{F}}\right)$.

Upon reaching the degradation level $\varepsilon$, an intervention is executed after $t_{\mathrm{p}}$ time-units, unless a failure occurs during the planning period $t_{\mathrm{p}}$. In other words, the intervention is a CBM-intervention if there is no failure, i.e., if $t_{\varepsilon, \mathrm{F}} \geq t_{\mathrm{p}}$, and it is a $\mathrm{CM}$ if a failure occurs, i.e., if $t_{\varepsilon, \mathrm{F}}<t_{\mathrm{p}}$. Hence, $\mathbb{P}\{\mathrm{CBM}\}$ is given by the cumulative reliability probability $R^{\prime \prime}\left(t_{\mathrm{p}}\right)$, defining the probability of $t_{\varepsilon, \mathrm{F}} \geq t_{\mathrm{p}}$. Similarly, $\mathbb{P}\{\mathrm{CM}\}$ is defined by the cumulative failure probability $F^{\prime \prime}\left(t_{\mathrm{p}}\right)$. Using Eqs. (2-3) and (8), we thus find:

$$
\begin{gathered}
\mathbb{P}\{\mathrm{CBM}\}=\exp \left[-\left(\frac{t_{\mathrm{p}}}{(1-\varepsilon) \alpha}\right)^{\beta}\right], \text { and } \\
\mathbb{P}\{\mathrm{CM}\}=1-\exp \left[-\left(\frac{t_{\mathrm{p}}}{(1-\varepsilon) \alpha}\right)^{\beta}\right] .
\end{gathered}
$$

To determine the expected maintenance cycle time $\mathbb{E}\left\{T_{\mathrm{c}}\right\}$, we make a distinction between the expected time to degrade from replacement until the intervention threshold $\varepsilon$, and the expected time to degrade from $\varepsilon$ to the CBM or CM intervention, respectively. The first term, $\mathbb{E}\left\{t_{\varepsilon}\right\}$, can be found using (4) and (7). The second and third term can be found by multiplying the degradation time from $\varepsilon$ to the $\mathrm{CBM} / \mathrm{CM}$ intervention (i.e., $t_{\mathrm{p}}$ for a $\mathrm{CBM}$ intervention and $t_{\varepsilon, \mathrm{F}}$ for a $\mathrm{CM}$ intervention), by its respective probabilities, defined by Eqs. (9-10), which leads to $t_{\mathrm{p}} \times \mathbb{P}\{\mathrm{CBM}\}$ for a CBM intervention and $\int_{0}^{t_{\mathrm{p}}} t_{\varepsilon, \mathrm{F}} f^{\prime \prime}\left(t_{\varepsilon, \mathrm{F}}\right) \mathrm{d} t_{\varepsilon, \mathrm{F}}$ for a CM intervention. Hence we obtain:

$$
\begin{aligned}
\mathbb{E}\left\{T_{\mathrm{c}}\right\} & =\varepsilon \alpha \Gamma\left(1+\frac{1}{\beta}\right)+t_{\mathrm{p}} \exp \left[-\left(\frac{t_{\mathrm{p}}}{(1-\varepsilon) \alpha}\right)^{\beta}\right] \\
& +\int_{t_{\varepsilon, \mathrm{F}}=0}^{t_{\mathrm{p}}} t_{\varepsilon, \mathrm{F}} \frac{\beta}{(1-\varepsilon) \alpha}\left(\frac{t_{\varepsilon, \mathrm{F}}}{(1-\varepsilon) \alpha}\right)^{\beta-1} \exp \left[-\left(\frac{t_{\varepsilon, \mathrm{F}}}{(1-\varepsilon) \alpha}\right)^{\beta}\right] \mathrm{d} t_{\varepsilon, \mathrm{F}}
\end{aligned}
$$

Using Eqs. (9-11), the objective function under a preventive CBM policy can be analytically evaluated, apart from the expected on hand inventory, $\mathbb{E}\{I\}$, for which we need to rely on simulation. The values of $\varepsilon, s$ and $S$ are then optimized taking the stochastic character of the failure behaviour into account in the simulation.

As explained in Section 4.2, we can adjust the inventory policy under CBM, by exploiting the ADI that an upcoming maintenance intervention is announced as soon as the degradation level surpasses $\varepsilon$. The ADI then reduces the risk period $L_{\mathrm{S}}$ with the demand lead time $L_{\mathrm{D}} \leq t_{\mathrm{p}}$, and we reduce the inventory position as soon as its demand is announced by the anticipated maintenance action. Also the demand for corrective maintenance exhibits some advance demand information, as a failure will only happen after surpassing the intervention threshold. The value of $L_{\mathrm{D}}$ is then the time it takes to degrade from $\varepsilon$ until 
the CBM or CM intervention. This demand lead time is stochastic. Its expected value is given by the second and third term of Eq. (11):

$$
\mathbb{E}\left\{L_{\mathrm{D}}\right\}=t_{\mathrm{p}} \exp \left[-\left(\frac{t_{\mathrm{p}}}{(1-\varepsilon) \alpha}\right)^{\beta}\right]+\int_{t_{\varepsilon, \mathrm{F}=0}}^{t_{\mathrm{p}}} t_{\varepsilon, \mathrm{F}} \frac{\beta}{(1-\varepsilon) \alpha}\left(\frac{t_{\varepsilon, \mathrm{F}}}{(1-\varepsilon) \alpha}\right)^{\beta-1} \exp \left[-\left(\frac{t_{\varepsilon, \mathrm{F}}}{(1-\varepsilon) \alpha}\right)^{\beta}\right] \mathrm{d} t_{\varepsilon, \mathrm{F}}
$$

\section{Numerical Study}

In this section, we discuss the results of our numerical study analysing the impact of the maintenance policies on the spare part inventory requirements, with special attention to the impact of advance demand information (ADI) inherent to preventive maintenance. Our case company typically operates in the heavy-equipment manufacturing sector. The machines produced are capital intensive, having a long lifetime. We consider parameter values that are validated by our original equipment manufacturer in the compressed air industry. The machines produced contain of course a whole range of components, which differ in lifetime, component cost, failure, the risk of consequential damage and the failure behaviour. The components range from consumables, supporting consumables to expensive and vital components. For that reason, the parameter values correspond to a representative set of spare parts that are replaced during the lifetime of the machines. These spare parts vary in their failure behaviour and maintenance costs. We consider degradation and failure behaviour as stochastic, making it mathematically intractable to analytically derive the spare part inventory behaviour. We therefore perform an extensive simulation experiment and optimise the decision variables with the objective to minimise the expected cost function. However, due to the stochastic nature of the failure behaviour, the effective cost performance is stochastic. We display this stochasticity by providing the 25 th and 75 th percentiles of the cost performance measured in 10,000 simulation runs. For reasons of confidentiality we disguised some information, however without loss of generality. Indeed, the range of parameter values used is realistic and the reported percentage wise impact on inventories offers sufficient insights into the potential cost savings for companies operating in the capital intensive equipment manufacturing sector.

Given the focus of this paper on inventory requirements, in the section below we only report on the component's inventory related costs, $I C(P)$. It should be clear, however, that in our simulation experiment we explore the full model as given in (5). In other words, we also take the maintenance related costs, $M C(P)$, into account when optimising our decision variables. We rely on simulation mainly because we cannot derive analytically $\mathbb{E}\{I\}$, the expected on hand inventory, which is required to meet the desired fill rate, $F_{\mathrm{r}}$. As shown in section 4 , we are able to derive analytically the remaining variables of model (5). The analytical expressions in section 4 can be used to validate our simulation results.

\subsection{Design of the experiment}

Table 2 summarises the parameter values used in our numerical experiment. These parameter values are used to characterise different components: e.g., components with a short time to failure are represented by a value $\alpha=2,000$ versus components that fail less often are here characterised by $\alpha=20,000$; Components with a high downtime cost (or high consequential damage) in case of breakdown, will have a higher cost of corrective maintenance, etc. We perform a full factorial experiment, which means that 
Table 2: Setup of the numerical experiment

\begin{tabular}{ll}
\hline Parameter & values \\
\hline$M$ & $1 ; 10 ; 100$ \\
$R$ & 2,000 \\
$\alpha$ & 2,$000 ; 20,000$ \\
$\beta$ & $1.5 ; 3$ \\
$C_{\mathrm{h}}$ & 0.1 \\
$K$ & 1 \\
$C_{\mathrm{PM}}$ & 1 \\
$C_{\mathrm{CBM}}$ & $C_{\mathrm{PM}} ; 2 C_{\mathrm{PM}} ; 4 C_{\mathrm{PM}}$ \\
$C_{\mathrm{CM}}$ & $C_{\mathrm{CBM}} ; 2 C_{\mathrm{CBM}} ; 4 C_{\mathrm{CBM}}$ \\
$t_{\mathrm{p}}$ & $120 ; 240$ \\
$L_{\mathrm{S}}$ & $240 ; 480$ \\
$F_{\mathrm{r}}$ & 0.99 \\
\hline
\end{tabular}

we consider $3 \times 1 \times 2 \times 2 \times 1 \times 1 \times 1 \times 3 \times 3 \times 2 \times 2 \times 1=432$ experimental setups, corresponding to all parameter value combinations in Table 2 . For each set of parameter values we optimise the inventory policy parameters $(s, S)$ and the maintenance policy parameters ( $\tau$ and $\varepsilon$ for PM and CBM, respectively), as specified in optimisation problem $(O)$ using exhaustive simulation-optimisation. Our experiments are simulated in Arena 14.5 and we performed an exhaustive search over an extensive range of parameter values.

We assume that each machine in the installed base runs continuously 250 days per year, 8 hours per day, resulting in $2,000 \mathrm{RH}$ per machine per year. We install the machines over an initialisation period on a random basis to have a different age for each machine. The initialisation period is 20,000RH when a CM and CBM policy is in use (corresponding to the highest $\alpha$-value in our experiment), whereas it is $\tau$ when a PM policy is adopted in order to have an equal probability of a PM intervention at a random time in the infinite time-horizon. The total warm-up period including the initialisation period is $100,000 \mathrm{RH}$, after which results are collected during another 100,000RH. We simulated 10,000 runs for each combination of parameter values.

Given our inventory focus, the size of the installed base (IB) is important. A large number of papers in the maintenance literature considers only one machine, and the few papers that do incorporate inventory, usually consider a relatively small IB. We consider a small $(M=1)$, medium $(M=10)$, and large $(M=100)$ IB of independent machines. A larger IB will result in a higher spare part demand with a relatively lower demand uncertainty. This will impact our results.

We express all time values in running hours $(\mathrm{RH})$, so that, for instance, $t_{\mathrm{p}}=120 \mathrm{RH}$ corresponds to 15 workdays or 3 weeks and $L_{\mathrm{S}}=480 \mathrm{RH}$ means 60 workdays or 12 weeks.

\subsection{Inventory control without using advance demand information}

We first consider the general practice where companies manage the spare part inventory, independent of the type of maintenance intervention. In other words, they do not exploit the advance demand information that is inherent to preventive maintenance, and consider all spare part replacements as random and unplanned.

Compared to CM, preventive maintenance policies increase the demand for spare parts, as some remaining 
useful life of components is not used. This increases the inventory requirements. We denote this as the 'demand effect'. Figures 4-6 illustrate this demand effect for a selective set of parameter values (although all findings apply to our full factorial experiment, the Figures only illustrate a selective set of parameter values). They report how the expected yearly inventory related costs $I C(P)$, defined as the yearly inventory holding costs plus ordering costs, increase for PM and CBM compared to CM. We find that the demand effect is stronger for PM than for CBM, indicating that the number of interventions is higher for PM than for CBM (when both preventive policies are optimised under the same parameter setup), which means that on average more lifetime is wasted under PM. In our experiments we find that the inventory related costs under PM are on average between 20-84\% higher than under CM (the white boxes display the 25 th and 75 th percentile); in an identical parameter setup, the inventory related cost increase under CBM is only on average $2-19 \%$ compared to CM.

Insert Figures 4-6 about here.

We find that a lower expected time to failure (represented by lower $\alpha$-values) results in higher inventory cost increases for PM and CBM compared to its equivalent setting under CM. This is illustrated in Figure 4 for two different $\alpha$-values. In case of a short average time to failure, the optimal intervention threshold will be set lower if it wants to avoid failures. This increases the 'demand effect', and likewise the inventory requirements. This is also illustrated in Figure 5, which reports two settings with a different IB and failure behaviour, but with equal number of failures under CM. It confirms that the demand effect is stronger for a small number of machines that often fail (small $\alpha$-value) versus a large installed base of machines with components that have a long average time to failure, due to the lower intervention threshold when $\alpha$ is small.

Under CBM, we observe that the increase in inventory costs is dependent on the variance of the failure time (see Figures 5-6). Generally, for higher values of the variance of the failure time (represented by lower $\beta$-values) the inventory requirements increase under CBM. In those cases, all other things equal, the optimal policy either decreases the value of $\varepsilon^{*}$, or it will endure more failures. Both increase the demand for spare parts. Only when the components have a very long average time to failure (i.e., a large $\alpha$-value), the difference in inventory requirements under CBM and CM becomes negligible.

Furthermore, Figures 4 and 6 illustrate that the demand effect is stronger when the cost of a CM intervention increases compared to the cost of preventive maintenance, especially under PM. As $C_{\mathrm{CM}}$ increases, more preventive maintenance will be carried out to avoid failures by having lower values of $\tau^{*}$ or $\varepsilon^{*}$. This leads to higher spare part demand and hence higher inventory costs. This effect is more pronounced for small $\beta$-values, as more preventive maintenance will be carried out when the variance of the failure time is large.

Finally, we observe the impact of the installed base. As the IB grows, the total inventory related costs per machine decrease, indicating economies of scale due to a lower spare part demand variance and the pooling of orders, as shown in Figure 7 .

Insert Figure 7 about here. 


\subsection{Inventory requirements when leveraging advance demand information}

When we make use of the advance demand information, prevalent in preventive maintenance, in managing the inventory of spare parts, companies can reduce the risk period with a time span equivalent to the demand lead time. Remember that the demand lead time is equal to the supply lead time for the PM interventions' spare usage, and given by Eq. (12) for all spare parts under CBM. Based on the results of our numerical experiments we can conclude that this 'risk mitigation' effect indeed lowers the inventory related costs. The exact impact however depends on the PM or CBM maintenance policy.

Under a PM policy, inventory related costs are on average up to $15 \%$ lower compared to the case where we do not make use of ADI. But even by introducing ADI, the inventory related costs are still higher than under CM. Under a PM policy with ADI, the demand effect still dominates the risk mitigation effect. Under CBM however, we get a more promising result. Under a CBM policy, inventory related costs are on average up to $14 \%$ lower compared to the case where we do not make use of ADI. Even stronger, under CBM with ADI, inventory related costs can be lower than under the pure (non-ADI) CM policy. In other words, the risk mitigation effect dominates the demand effect in some cases. We only found this outcome for a CBM policy. This phenomenon is illustrated in Figure 8, for a specific parameter setting.

Insert Figure 8 about here.

In addition to the above mentioned general insight, we are also able to refine the insight along the following lines. First, our results indicate that the benefit of using ADI is stronger for slow degrading components (with a long expected lifetime, indicated by a large $\alpha$-value in its failure behaviour), as shown in Figure 9. In those cases, the demand uncertainty is large and a reduction of the risk period will have a stronger effect. In contrast, when we have a large demand with low uncertainty, risk mitigation will only have a marginal additional impact on inventory cost reductions. In Figure 10 we control for the number of failures (we report the same setting as in Figure 5 with different IB and failure behaviour, but with same number of failures under $\mathrm{CM}$ ). The results confirm that the value of ADI is stronger for slow degrading components (large $\alpha$-value). In those cases, less failures occur during the planning period $t_{\mathrm{p}}$, which increases the benefit of pre-announcing the upcoming spare part demand. Second, we find that the value of using ADI increases with the planning period $t_{\mathrm{p}}$. In case of a longer planning period, the spare part use is announced a longer time upfront, which increases the risk mitigation effect. This can be seen in Figure 9.

Insert Figures 9-10 about here.

\section{Conclusions}

In this paper we analyse, through simulation, the impact of jointly optimising the maintenance policy and the corresponding inventory related costs. It is clear that the maintenance policy has an impact on the 
behaviour of the required spare part inventory. Indeed, preventive maintenance policies increase the total demand for spare parts compared to corrective maintenance, since the former do not exploit the entire useful life of the components. This demand effect increases the spare part's inventory requirements. This drawback of preventive maintenance policies can be reduced and in some cases even eliminated due to the existence of advance demand information. Preventive maintenance policies exhibit advance demand information because the demand for a spare part is announced some time in advance of the actual spare part consumption. This time span is called the demand lead time. The existence of a demand lead time reduces the risk period. This is called the risk mitigation effect. Evaluating the inventory related costs of a maintenance policy comes down to comparing the demand effect with the risk mitigation effect. As is shown in this paper, this evaluation depends on several factors: the type of preventive policy (periodic maintenance versus condition-based maintenance), the size of the installed base, the failure characteristics of the components and the underlying cost structure and operational characteristics of the maintenance policies. Our full factorial experiment is based on a realistic setting of an OEM in the compressed air industry with whom we collaborate. For each experiment we optimise the inventory policy parameters $(s, S)$ and the maintenance policies (the length of the maintenance interval $\tau^{*}$ and the intervention threshold $\varepsilon^{*}$, for respectively PM and CBM). In our numerical experiment, we find that that the demand effect is stronger for PM than for CBM, which means that on average more lifetime is wasted under the optimised PM policy than under the optimised CBM policy. We further find that the demand effect can to a large extent be offset using advance demand information in managing the spare part inventory; for condition-based maintenance, we find that inventories can even be lower compared to corrective maintenance, i.e., the risk mitigation effect is larger than the demand effect. For the OEM with whom we work, our analysis sheds light on the behaviour of the inventory related costs under various maintenance policies.

\section{References}

[1] Barlow, R. and Hunter, L. (1960). Optimum preventive maintenance policies. Operations Research, 8(1):90-100.

[2] Basten, R. J. I. and Van Houtum, G.-J. (2014). System-oriented inventory models for spare parts. Surveys in operations research and management science, 19(1):34-55.

[3] Bijvank, M. and Vis, I. F. A. (2011). Lost-sales inventory theory: A review. European Journal of Operational Research, 215(1):1-13.

[4] Camci, F. (2009). System maintenance scheduling with prognostics information using genetic algorithm. IEEE Transactions on Reliability, 58(3):539-552.

[5] Christer, A. H. (1982). Modelling inspection policies for building maintenance. The Journal of the Operational Research Society, 33(8):723-732.

[6] Christer, A. H. and Waller, W. M. (1984). Delay time models of industrial inspection maintenance problems. The Journal of the Operational Research Society, 35(5):401-406.

[7] Deshpande, V., Iyer, A. V., and Cho, R. (2006). Efficient supply chain management at the U.S. Coast Guard using part-age dependent supply replenishment policies. Operations Research, 54(6):1028-1040.

[8] Donders, J. (2009). Logistieke uitdagingen bij windmolenparken in zee. Logistiek.nl. In Dutch. 
[9] Gallego, G. and Özer, O. (2001). Integrating replenishment decisions with advance demand information. Management Science, 47(10):1344-1360.

[10] Godoy, D. R., Pascual, R., and Knights, P. (2013). Critical spare parts ordering decisions using conditional reliability and stochastic lead time. Reliability Engineering \& System Safety, 119:199-206.

[11] Goossens, A. J. M. and Basten, R. J. I. (2015). Exploring maintenance policy selection using the analytic hierarchy process; an application for naval ships. Reliability Engineering 83 System Safety, 142:31-41.

[12] Hariharan, R. and Zipkin, P. (1995). Customer-order information, leadtimes, and inventories. Management Science, 41(10):1599-1607.

[13] Hill, R. (1999). Bayesian decision-making in inventory modelling. IMA Journal of Mathematics Applied in Business and Industry, 10(2):165-176.

[14] Jardine, A. K. S. and Tsang, A. H. C. (2006). Maintenance, Replacement, and Reliability. Theory and Applications. Dekker Mechanical Engineering. CRC Press, Boca Raton (FL).

[15] Jones, J. (2006). Integrated logistics support handbook. McGraw-Hill, New York (NY, USA), third edition edition.

[16] Karsten, F. and Basten, R. J. (2014). Pooling of spare parts between multiple users: How to share the benefits? European Journal of Operational Research, 233(1):94-104.

[17] Li, R. and Ryan, J. K. (2011). A Bayesian inventory model using real-time condition monitoring information. Production and Operations Management, 20(5):754-771.

[18] Louit, D., Pascual, R., Banjevic, D., and Jardine, A. K. S. (2011). Condition-based spares ordering for critical components. Mechanical Systems and Signal Processing, 25:1837-1848.

[19] Lu, C. J. and Meeker, W. O. (1993). Using degradation measures to estimate a time-to-failure distribution. Technometrics, 35(2):161-174.

[20] Muckstadt, J. A. (2005). Analysis and Algorithms for Service Parts Supply Chains. Springer, New York (NY).

[21] Pintelon, L. and Van Puyvelde, F. (2006). Maintenance Decision Making. Acco, Leuven (Belgium).

[22] Poppe, J., Boute, R., and Lambrecht, M. (2016). A hybrid condition-based maintenance policy for continuously monitored components with two degradation thresholds. KU Leuven, KBI working paper 1609 .

[23] Sarker, R. and Haque, A. (2000). Optimization of maintenance and spare provisioning policy using simulation. Applied Mathematical Modelling, 24(10):751-760.

[24] Sherbrooke, C. C. (2004). Optimal inventory modelling of systems. Multi-echelon techniques. Kluwer, Dordrecht (The Netherlands), second edition.

[25] Srinivasan, R. and Parlikad, A. K. (2013). Value of condition monitoring in infrastructure maintenance. Computers \&3 Industrial Engineering, 66(2):233-241. 
[26] Thomas, L. C. and Osaki, S. (1978). An optimal ordering policy for a spare unit with lead time. European Journal of Operational Research, 2(6):409-419.

[27] Tinga, T. (2010). Application of physical failure models to enable usage and load based maintenance. Reliability Engineering and System Safety, 95:1061-1075.

[28] Tinga, T. (2013). Principles of Loads and Failure Mechanisms: Applications in Maintenance, Reliability and Design. Springer Science \& Business Media.

[29] Topan, E., Tan, T., Van Houtum, G. J., and Dekker, R. (2016). Using imperfect advance demand information in lost-sales inventory systems. BETA Working paper, 512.

[30] Van Dongen, L. A. M. (2011). Maintenance engineering: Maintaining links. Inaugural lecture, University of Twente.

[31] Van Horenbeek, A. and Pintelon, L. (2013). A dynamic predictive maintenance policy for complex multi-component systems. Reliability Engineering and System Safety, 120:39-50.

[32] Van Houtum, G. J. and Kranenburg, A. A. (2015). Spare Parts Inventory Control under System Availability Constraints. International Series in Operations Research \& Management Science. Springer.

[33] Van Noortwijk, J. M. (2009). A survey of the application of gamma processes in maintenance. Reliability Engineering \&3 System Safety, 94:2-21.

[34] Van Horenbeek, A., Buré, J., Cattrysse, D., Pintelon, L., and Vansteenwegen, P. (2013). Joint maintenance and inventory optimization systems: A review. International Journal of Production Economics, 143(2):499-508.

[35] Wang, H. (2002). A survey of maintenance policies of deteriorating systems. European Journal of Operational Research, 139:469-489.

[36] Wang, W. (2008). Delay Time Modelling, pages 345-370. Springer London, London.

[37] Zohrul Kabir, A. and Al-Olayan, A. S. (1996). A stocking policy for spare part provisioning under age based preventive replacement. European Journal of Operational Research, 90(1):171-181. 


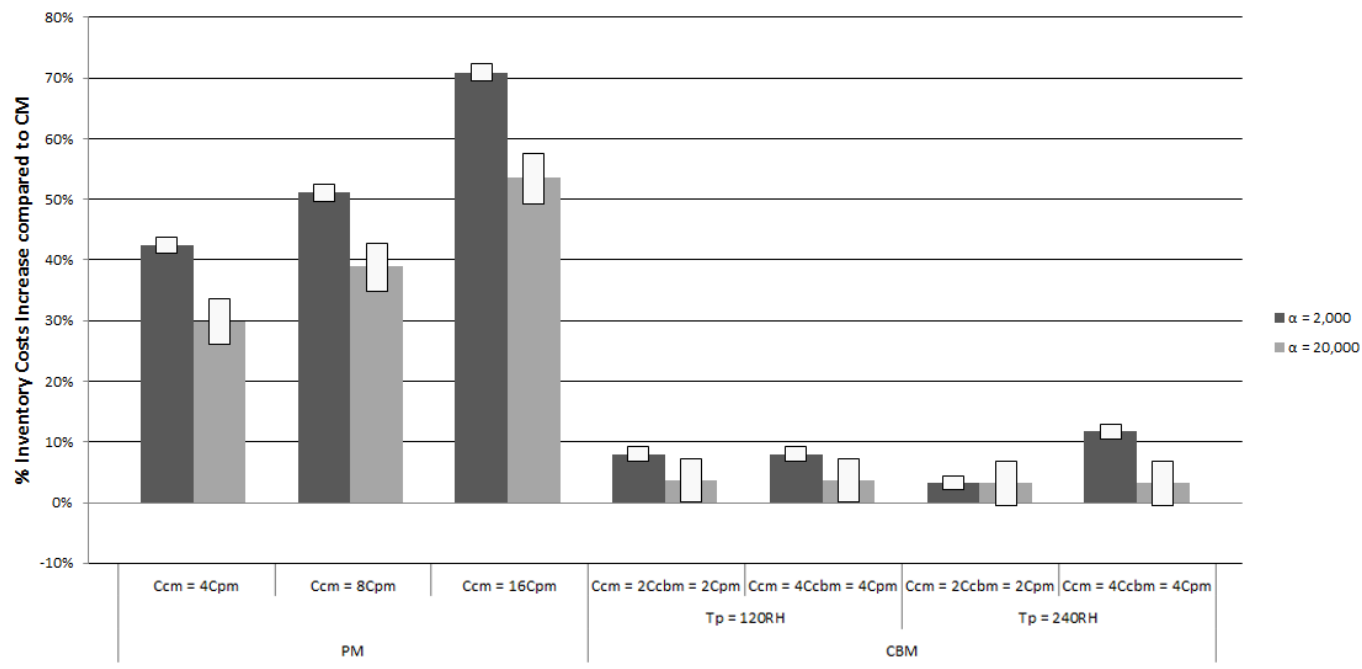

Figure 4: Inventory related cost increase of preventive maintenance compared to corrective maintenance, without using ADI; $\beta=3, L_{\mathrm{S}}=240 \mathrm{RH}, M=10$ and varying values of $\alpha, t_{\mathrm{p}}$, and intervention costs.

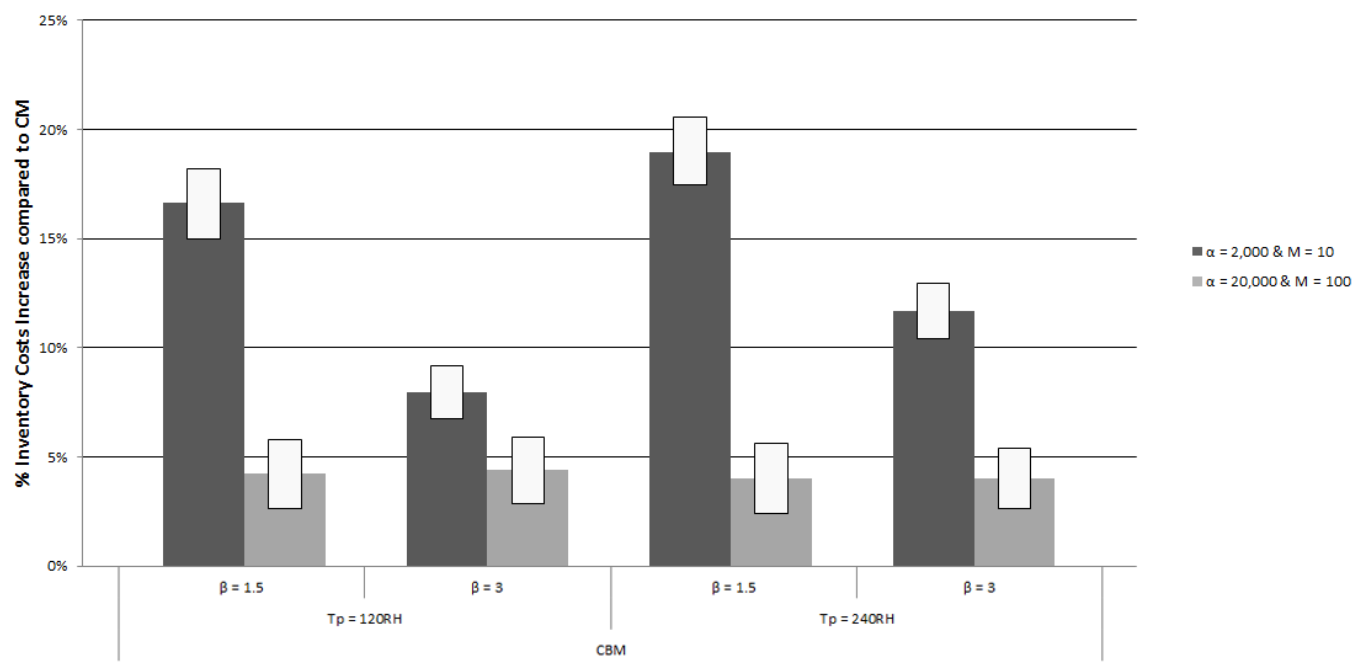

Figure 5: Inventory related cost increase of preventive maintenance compared to corrective maintenance without using ADI, for $\alpha=2,000$ and $M=10$ vs. $\alpha=20,000$ and $M=100 ; L_{\mathrm{S}}=240 \mathrm{RH}, C_{\mathrm{CM}}=$ $4 C_{\mathrm{CBM}}=16 C_{\mathrm{PM}}$ and varying values of $\beta$ and $t_{\mathrm{p}}$. 


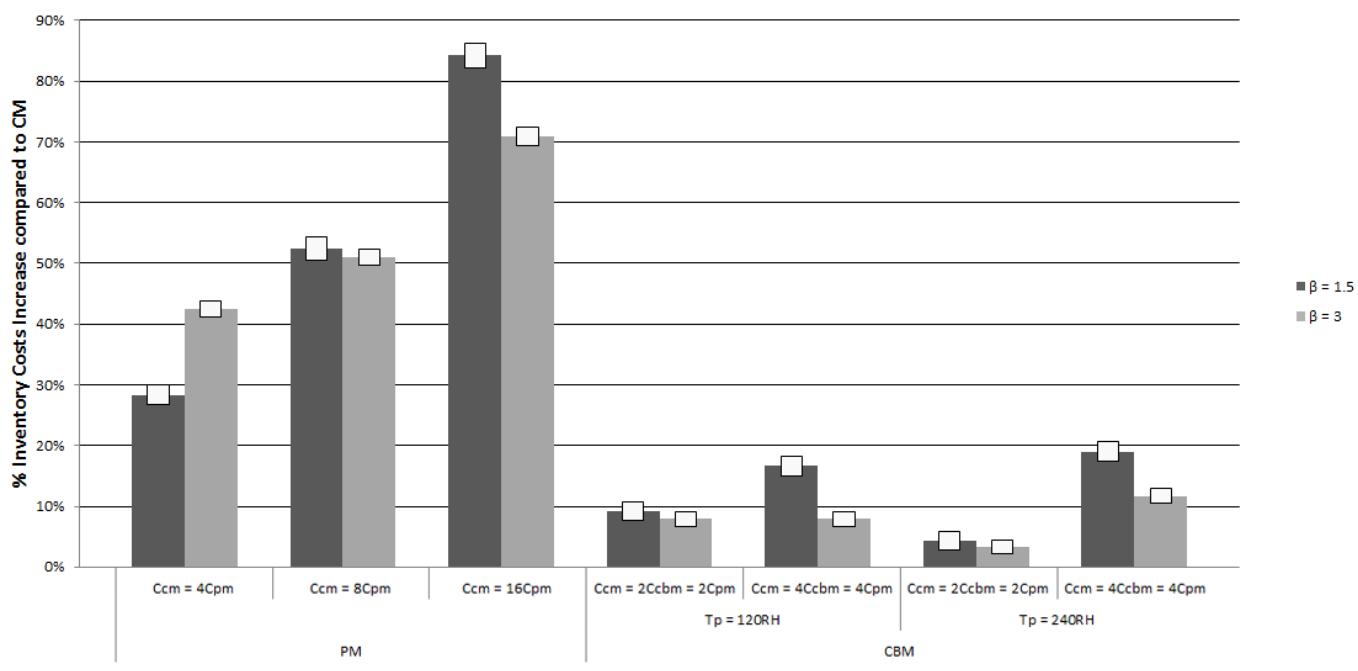

Figure 6: Inventory related cost increase of preventive maintenance compared to corrective maintenance, without using ADI; $\alpha=2,000, L_{\mathrm{S}}=240 \mathrm{RH}, M=10$ and varying values of $\beta, t_{\mathrm{p}}$, and intervention costs.

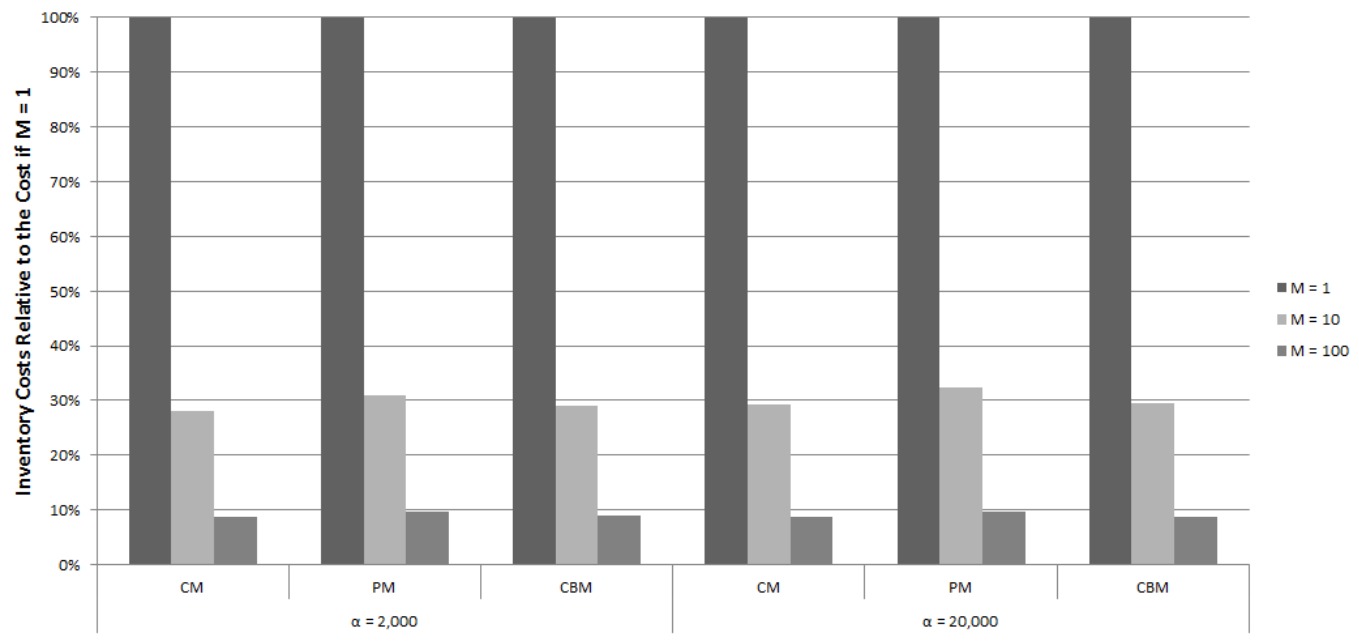

Figure 7: Inventory costs per machine relative to the costs under $M=1$, without using ADI; $\beta=1.5$, $t_{\mathrm{p}}=240 \mathrm{RH}, L_{\mathrm{S}}=480 \mathrm{RH}, C_{\mathrm{CM}}=4 C_{\mathrm{CBM}}=16 C_{\mathrm{PM}}$ and varying values of $\alpha$ and $M$. 


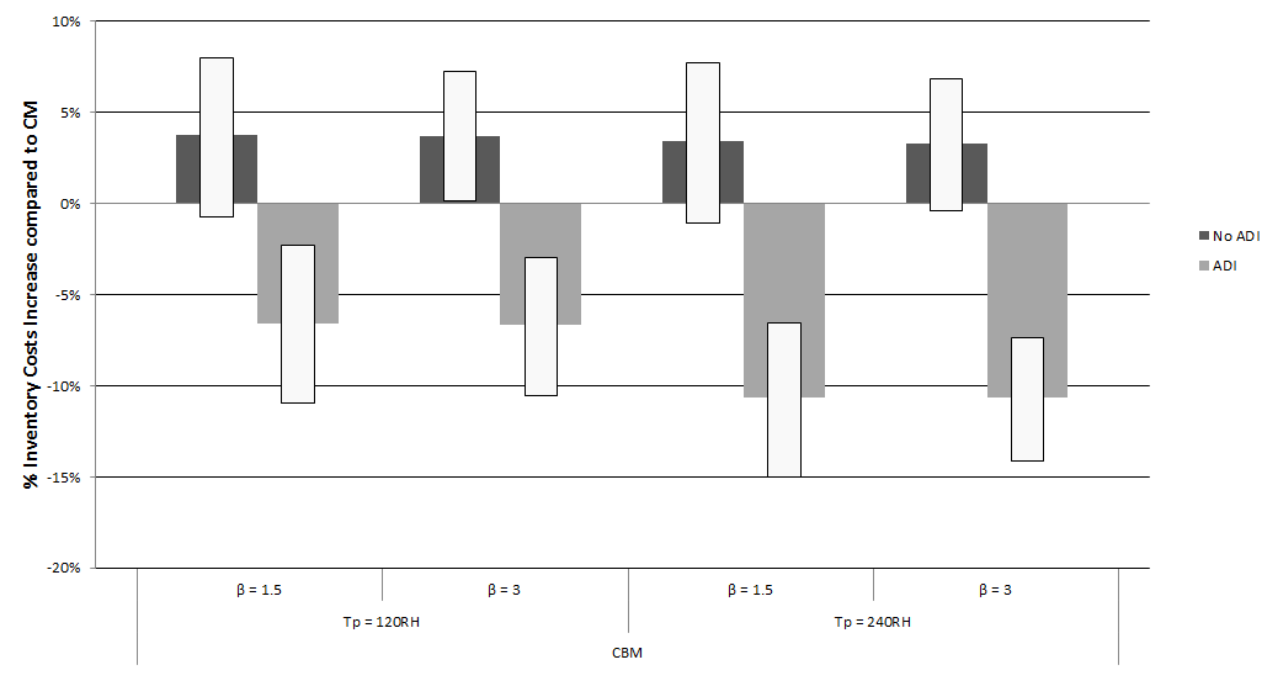

Figure 8: Inventory related costs of CBM compared to CM, with and without using ADI; $L_{\mathrm{S}}=240 \mathrm{RH}$, $M=10, C_{\mathrm{CM}}=2 C_{\mathrm{CBM}}=2 C_{\mathrm{PM}}, \alpha=20,000$ and varying values of $\beta$ and $t_{\mathrm{p}}$

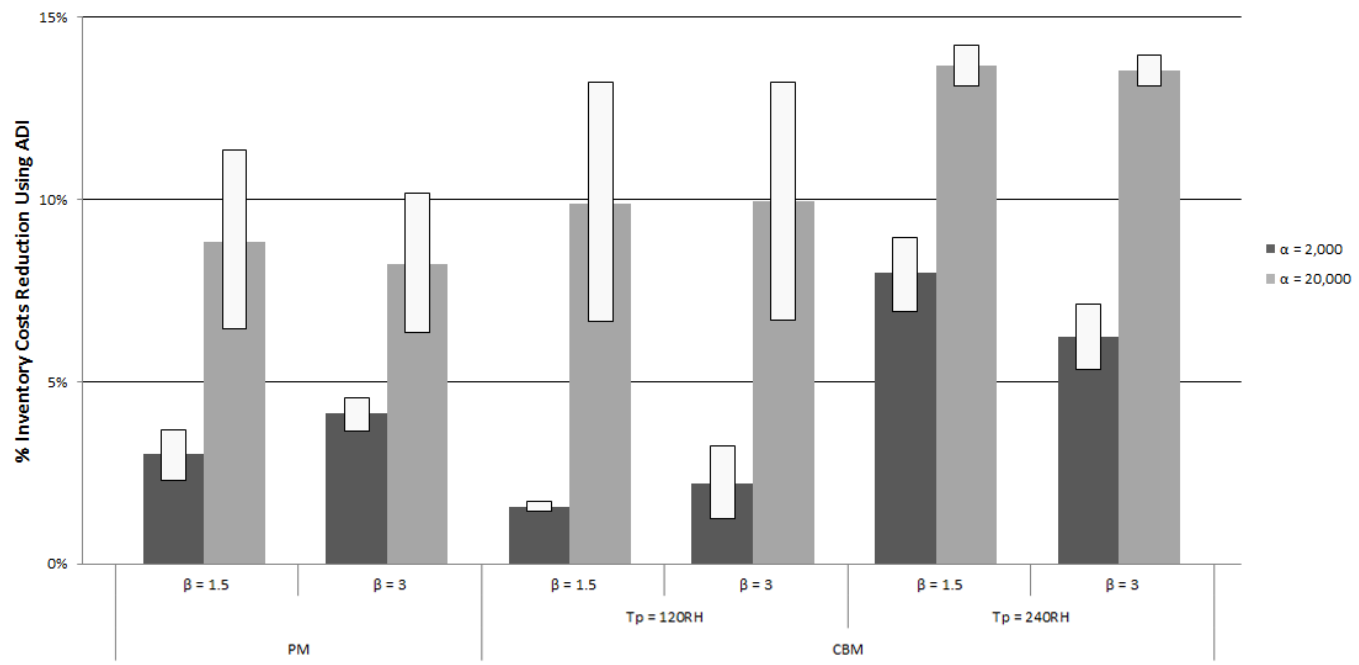

Figure 9: Reduction in inventory related costs when using ADI in preventive maintenance compared to not using ADI; $L_{\mathrm{S}}=240 \mathrm{RH}, M=10, C_{\mathrm{CM}}=2 C_{\mathrm{CBM}}=8 C_{\mathrm{PM}}$ and varying values of $\alpha, \beta$ and $t_{\mathrm{p}}$. 


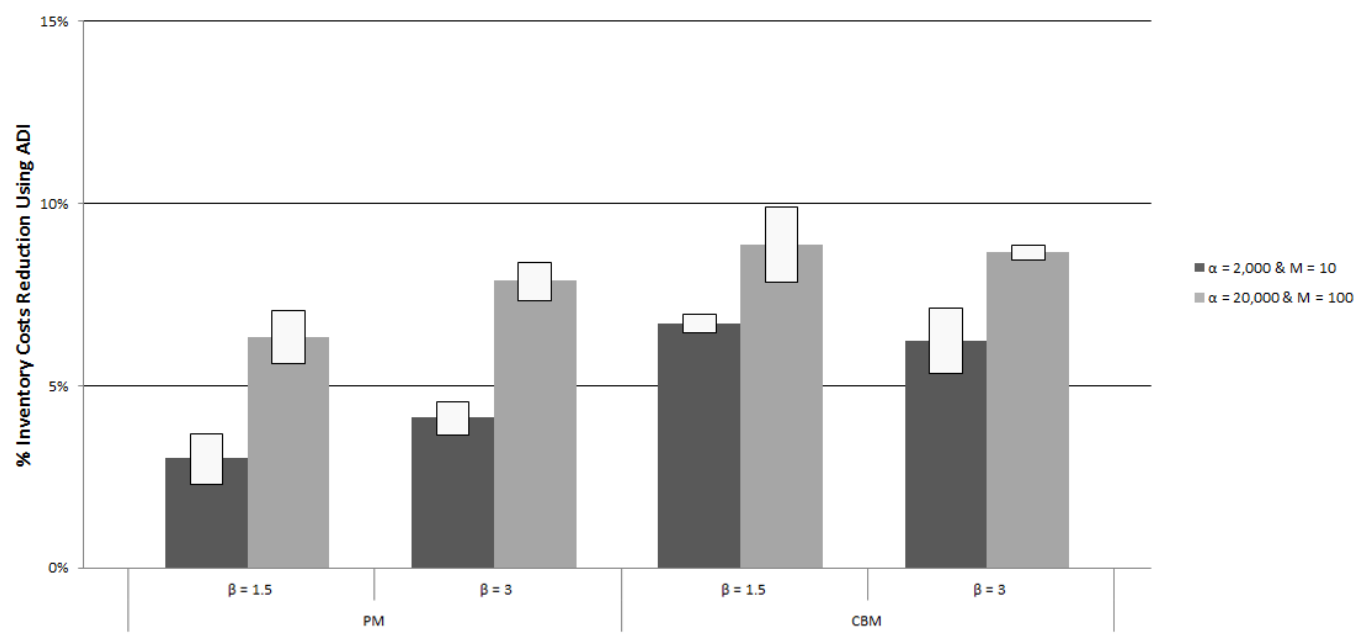

Figure 10: Reduction in inventory related costs when using ADI compared to not using ADI, for $\alpha=$ 2,000 and $M=10$ vs. $\alpha=20,000$ and $M=100 ; L_{\mathrm{S}}=240 \mathrm{RH}, t_{\mathrm{p}}=240 \mathrm{RH}, C_{\mathrm{CM}}=4 C_{\mathrm{CBM}}=16 C_{\mathrm{PM}}$ and varying values of $\beta$. 